\title{
Using photon mapping for multispectral modeling of scenes within the THz range
}

\author{
Wojciech Gołębiowski* \\ SKA Polska Sp. z o.o., al. Jerozolimskie 125/127 p. 406, 02-017 Warszawa
}

Received August 06, 2012; accepted September 24, 2012; published September 30, 2012

\begin{abstract}
A method for simulating the appearance of a large, geometrically modeled environment in the $\mathrm{THz}$ range is presented. A photon mapping technique is used with Monte Carlo photon sampling. The propagation of radiation within the scene is simulated for multiple discrete radiation frequencies, various materials are being modeled with emissivity, reflectivity and attenuation characteristics. The results are used to predict the answer of a passive, multispectral terahertz detector matrix.
\end{abstract}

The recent development of a passive spectroscopic detector working in the $\mathrm{THz}$ range [1] opens up possibilities for remote identification of certain materials which have spectral features within this range. Because of the passive nature of a measured signal such identification requires special processing techniques to distinguish between a subject and the background. The described method was developed to allow testing of various approaches for multispectral, passive image processing in the $\mathrm{THz}$ range, particularly for testing the efficiency of remote material recognition algorithms in various geometric configurations. The second important goal is allowing to optimize the set of discrete sensitivity frequencies for the sensor, taking into account a certain set of materials to be recognized as well as the influence of the atmosphere (because of contamination of water vapour air has a strongly nonlinear spectral attenuation characteristics in the $\mathrm{THz}$ range with significant radiation blocking and passing areas).

In order to realize above goals the following assumptions and simplifications are taken into consideration:

- Simulation for multiple narrowband ranges (to mimic the sensor)

- Energy conservation is obeyed for each frequency independently (no phosphorescence or fluorescence)

- Plank's Law - the amount of radiation emitted by a certain body is dependent on its temperature, area, radiation frequency and emissivity characteristics

- Other material features are spectral characteristics of reflectivity and transmissivity (modelled as a linear attenuation coefficient)

- Lambertian emission and reflection models are used (ideally diffusive)

"E-mail: w.golebiowski@ska-polska.pl
- Definable atmosphere attenuation characteristics, atmosphere itself doesn't emit radiation

- Phenomena like refraction, diffraction, interference are not modelled (radiation propagates along straight lines)

The described method is a global illumination algorithm based on a photon mapping method [2]. The simulated scene is modelled as a set of objects (Fig. 1). There are two types of objects: solid and shell with a defined thickness (to simulate the propagation of radiation through relatively thin covers). Objects are approximated as a set of triangles which may form an open or closed surface. Each of the objects has its assigned type (solid or shell), temperature, thickness and material. The materials are defined with an emissivity, reflectivity and linear attenuation coefficient for each of the declared frequencies. A set of frequencies to be simulated is declared together with a relative spectral thickness (assuming the Gaussian distribution within a frequency domain).

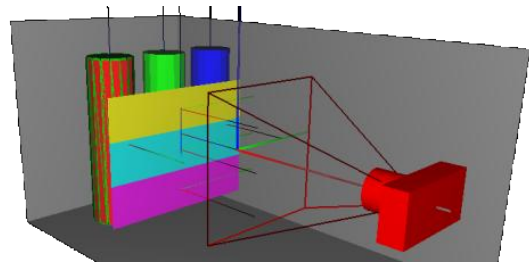

Fig. 1. Definition of the scene with GUI.

As a starting point for calculation a so-called rendering equation [3] is used. In terms of the above assumptions, the radiation leaving an elementary surface for each of the frequencies may be described as:

$$
L_{O}=L_{e}+L_{R}+L_{T}
$$

where the indices denote respectively: outgoing radiation, radiation emitted by the surface, radiation reflected from the surface and radiation transmitted through the surface. These terms may be expressed as following (for a given outgoing direction):

$$
\begin{aligned}
& L_{e}=E I_{o}(T, v)\left(\vec{\omega}_{o} \cdot \vec{\omega}_{n}\right) \\
& L_{R}=\int_{\Omega_{+}} f_{R}\left(\vec{\omega}_{o}, \vec{\omega}_{i}\right) L_{i}\left(\vec{\omega}_{i}\right)\left(-\vec{\omega}_{i} \cdot \vec{\omega}_{n}\right) d \vec{\omega}_{i} \\
& L_{T}=\int_{\Omega_{-}} f_{T}\left(\vec{\omega}_{o}, \vec{\omega}_{i}\right) L_{i}\left(\vec{\omega}_{i}\right)\left(\vec{\omega}_{i} \cdot \vec{\omega}_{n}\right) d \vec{\omega}_{i}
\end{aligned}
$$


$\vec{\omega}_{o}, \vec{\omega}_{i}, \vec{\omega}_{n}-$ outgoing, incoming and surface normal directions;

$E-$ emissivity factor for given frequency,

$I_{o}(T, v)$ - black body radiation;

$\Omega_{+}, \Omega_{-}-$positive and negative hemispheres;

$L_{i}$ - incoming radiation;

$f_{R}$ - so-called BRDF (Bidirectional Reflection

Distribution Function;

$f_{T}-$ BTDF (Bidirectional Transmission Distribution

Function;

$E, f_{R}, f_{T}$ - properties of material, where the latter two contain information about reflectivity and transmissivity. These functions must be properly normalized in order to conserve energy. In the presented method a Lambertian reflection model is used as it seems to approximate the refection phenomenon in the $\mathrm{THz}$ range accurately enough for most of the materials of interest. However, any model may be used for this purpose as long as it is normalized and supports the importance sampling for Monte Carlo ray tracing (i.e. Ward's anisotropic model [4]).

Calculation is divided into two phases: creating a photon map (the map of incoming radiation along all the surfaces within a simulated environment) and rendering (estimating an image as seen by the sensor).

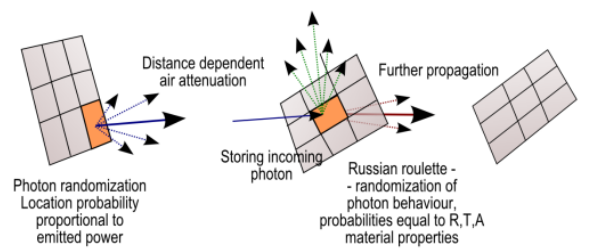

Fig. 2. Scheme of virtual photon propagation.

The photon map is created by random generation of the start point and the direction of virtual photon and by calculating its propagation through the scene (Fig. 2). The probability that a photon of a given frequency $v$ will be fired from a certain elementary surface is weighted by the amount of energy this surface emits and it may be expressed as:

where:

$$
p_{i}=\frac{E_{i} A_{i} I_{o}\left(T_{i}, v\right)}{\sum_{k=1}^{N} E_{k} A_{k} I_{o}\left(T_{k}, v\right)}
$$

$A_{i}$ - area of the surface;

$T_{i}$ - temperature of the object surface;

$N$ - number of all the surfaces.

The probability of firing the photon at a given off-normal direction:

$$
p(\varphi)=\cos \varphi
$$

Probabilities for the second angular component of the direction and location within the surface have uniform distribution. The power of the fired virtual photon is considered to be 1 .

Once the photon is randomized, it is being propagated through the scene. The hit position (elementary surface and location within it) is being found using a kd-tree search algorithm (for space sorting) and line-triangle intersection algorithms. If atmosphere attenuation is used, photon power is decreased using the following relation:

where:

$$
L=L_{0} e^{-\alpha(v) d}
$$

$L_{0}$ - initial power of photon;

$\alpha(v)$ - linear attenuation coefficient of atmosphere;

$d$ - photon travel distance.

Typically for $\alpha(v)$ HITRAN 2004 database is used [5], but an arbitrary spectral attenuation characteristics may be used as well.

At this point the photon may be either absorbed by the object, transmitted through it or reflected according to the material of a hit object. The behavior is determined with a so-called Russian roulette rule [6]. The following probabilities are used:

$$
\begin{aligned}
& p_{R}=R(v) \\
& p_{T}=\min \left(e^{-\alpha_{M}(v) D}, 1-p_{R}\right) \\
& p_{A}=1-p_{R}-p_{T}
\end{aligned}
$$

where:

$p_{R}, p_{T}, p_{A}-$ probabilities of photon reflection, transmission and absorption respectively;

$R(v)$ - frequency dependent reflectivity of a hit material; $\alpha_{M}(v)$ - frequency dependent linear attenuation coefficient of a hit material;

$D$ - thickness of a hit object along the photon direction.

In the case of reflection or incoming transmission direction, hit point and power of the photon are stored in the photon map (Fig. 3) and the photon is propagated further using the same rules. The propagation direction is randomized according to BRDF and BTDF models used. The process continues until the photon is absorbed or its power reaches a defined fraction of its initial power (i.e. 0.01 ) and then the photon is randomized and propagated. A photon firing phase is performed for a defined (configurable) number of photons to be fired.

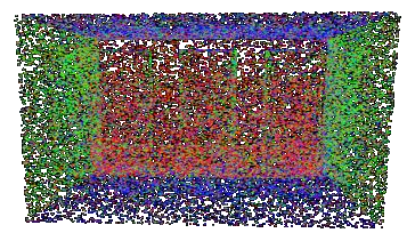

Fig. 3. Photon map visualization for scene from Fig. 1. Color indicate direction of incoming photon.

The next phase - rendering - is to calculate how the illuminated scene would be seen by a multispectral sensor. The sensor is defined with its position, looking direction, up direction, angle of view, aperture and resolution (the number of multispectral pixels). For each of the pixels a ray is cast in its viewing direction to localize the area within the scene it is looking at (Fig. 4). From this point a search within the photon map is performed in order to find photons which belong to the same surface as a central point and which are not further 
from it than a certain distance (coming from the angle of view of the pixel and relative orientation of the surface). The following formula is used to calculate the power measured by a single sensor for a single frequency:

$$
\begin{aligned}
& P_{M}=\left(P_{E}+P_{R, T}\right) \Omega_{S} A_{S} e^{-\alpha(v) d} \\
& P_{E}=E I_{o}(T, v)\left|\vec{\omega}_{S} \cdot \vec{\omega}_{n}\right| \\
& P_{R, T}=\frac{P_{A} \sum_{i=1}^{N} P_{i} f_{R, T}\left(\vec{\omega}_{S}, \vec{\omega}_{i}\right)\left|\vec{\omega}_{i} \cdot \vec{\omega}_{n}\right|}{K \pi r_{N}{ }^{2}}
\end{aligned}
$$

where:

$P_{E}$ - power emitted directly into the sensor's direction;

$P_{R, T}$ - indirect illumination (reflected and transmitted);

$\vec{\omega}_{S}-$ direction from a particular pixel;

$\vec{\omega}_{i}-$ single photon direction;

$P_{A}-$ sum of powers emitted from all surfaces;

$P_{i}$ - virtual power of a single photon $(0 . .1)$;

$N$ - number of photons found in a single query;

$f_{R, T}-$ BRDF or BTDF function (depending on a particular photon type);

$K$ - number of photons fired for a given frequency $v$;

$r_{N}$ - radius of area surrounding all photons found;

$\Omega_{S}$ - solid angle of a single pixel of the sensor;

$A_{S}$ - aperture of the sensor.

This procedure is repeated for all of the sensor's pixels and all defined frequencies until the whole multispectral image of the scene is created.

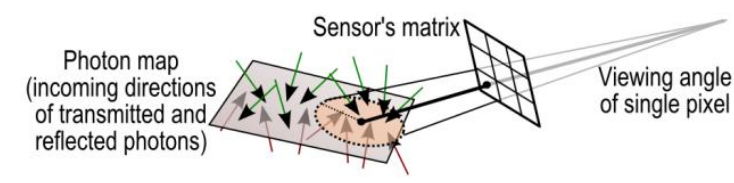

Fig. 4. Scheme of rendering phase.

As the search for photons is performed only within one object (to speed up the search and to avoid geometry analysis for objects that are placed very close to each other) some artifacts may appear close to borders of the objects or areas where surface normals change rapidly (edges). When it is relevant respective oversampling may be used (using several pixels for each physical pixel of the sensor) together with proper averaging.

During the rendering phase other information is created as well: visual representation, temperature map and distance map. Information from these so called virtual sensors is used to support further analysis of the $\mathrm{THz}$ multispectral image.

The nature of a photon mapping technique allows to repeat the rendering phase (i.e. for different points of view) without repeating a photon firing phase (which is more computationally expensive).

Graphical pre- and postprocessors were developed with a batch processing capability and support to calculate sequential scenes ( $\mathrm{THz}$ movies).

To illustrate the presented method the following test case was prepared. The scene (showed in Fig. 1) is composed of 3 cylinders of $0.75 \mathrm{~m}$ height, made of different artificial materials. They are covered with 3 stripes $0.1,0.5$ and $1 \mathrm{~mm}$ thick (from bottom to top respectively) made of a material with the attenuation coefficient showed in Fig 5.

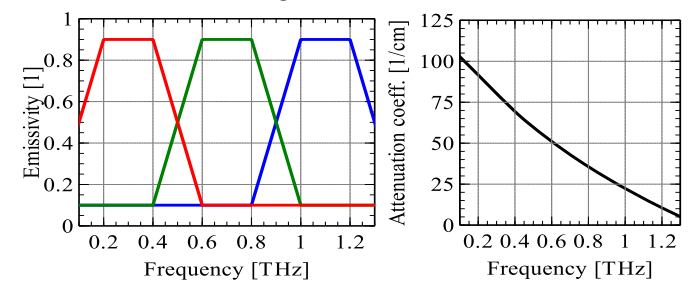

Fig. 5. Emissivity spectra for test case cylinders (left) and attenuation coefficient of a cover material. Colors correspond to colors from Fig. 1.

The sensor is located $1.5 \mathrm{~m}$ from cylinders and tuned for 6 evenly spaced frequencies from 0.2 to $1.2 \mathrm{THz}$ with a relative spectral thickness of $1 \%$. Air attenuation is turned off. Everything is enclosed in a $1 \mathrm{~m} \times 1 \mathrm{~m} \times 2 \mathrm{~m}$ box with a flat spectral characteristics. Temperatures of all objects are set to be around room temperature. Simulation was performed for 10 millions of photons and $64 \times 64$ sensor matrix. The results are presented in Fig. 7.

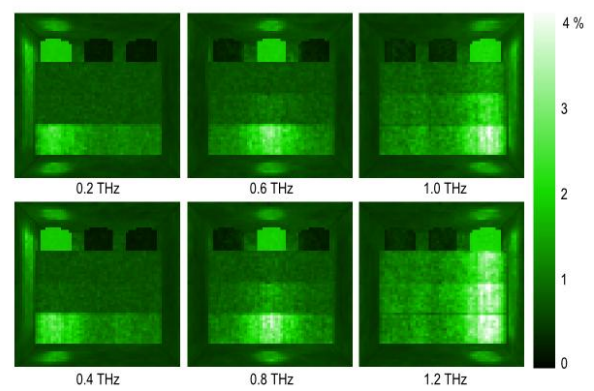

Fig. 6. Results of test case simulation normalized against black body radiation.

Although the method is adapted from computer graphics techniques (visual range with a limited number of light sources), it can be seen that it is usable in the $\mathrm{THz}$ range, where every object is considered to be a light source (in room temperature). The method was used within an optimization process to select optimal frequencies for a real sensor in order to be able to recognize selected dangerous materials.

The work has been done within FP6 European project Teraeye.

\section{References}

[1] M. Dell'Anna, V. Antonov, D. Bagliani, M. Biasotti, J. L. Coutaz, F. Gatti, M. Kiviranta, S. Kubatkin, E. Otto and M. Sypek, J. Low Temp. Phys. 167(3-4), 467 (2012).

[2] H.W. Jensen, Global Illumination using Photon Maps, Rendering Techniques '96, 21-30 (1996)

[3] J.T. Kajiya, The Rendering Equation, SIGGRAPH '86 Proceedings, 143-150 (1986).

[4] G. Ward, Measuring and Modeling Anisotropic Reflection, SIGGRAPH '92 Proceedings, 265-272, (1992)

[5] The HITRAN Database, http://www.cfa.harvard.edu/hitran/

[6] H. Kahn, Use of Different Monte Carlo Sampling Technique (Rand Corporation, 1955) 